\title{
Capital social et gestion du risque dans les réseaux de l'entrepreneuriat immigré en Afrique centrale : le cas des Maliens au Congo-Brazzaville ${ }^{1}$
}

\author{
Théophile Dzaka-Kikouta ${ }^{a}$, Luyindula G. Davy Makany ${ }^{b}$
}

\begin{abstract}
RÉSUMÉ. Partant de la littérature sur l'entrepreneuriat ethnique et d'une enquête effectuée en 2010, à Brazzaville, auprès de 220 répondants parmi les entrepreneurs immigrants maliens, cet article cherche à savoir dans quelle mesure les performances entrepreneuriales d'immigrants maliens exerçant au Congo-Brazzaville, dans des PME familiales, procèdent-elles du capital social? Celui-ci signifie un fort degré de réseautage des ressources ethniques selon Menzies, Brenner et Filion (2003). Nous émettons l'hypothèse que ces entrepreneurs, implantés au Congo depuis les années 1960, forment une «minorité intermédiaire durable » (Bonacich, 1973). L'étude identifie les facteurs de succès de cet entrepreneuriat en lien avec la gestion du risque par le recours au capital social induisant une réduction des coûts de transaction. L'impact de ces entrepreneurs sur le développement local du pays d'origine et du pays d'accueil est évalué; sont dégagées aussi leurs stratégies d'adaptation au risque d'éviction du marché, à cause de la concurrence récente des réseaux marchands d'immigrants chinois.
\end{abstract}

ABSTRACT. Based on ethnic entrepreneurship literature and a survey conducted in 2010, in Brazzaville, among 220 respondents among Malian immigrant entrepreneurs, this article looks at the extent to which the entrepreneurial performance of Malian immigrants, operating in Congo, via family SMEs do they proceed from social capital? This means a high degree of networking of ethnic resources according to Menzies, Brenner and Filion (2003). Our hypothesis is that these entrepreneurs, operating in the Congo since the 1960s, form "a sustainablemiddleman minority" (Bonacich, 1973). The study elucidates the factors of success of this entrepreneurship in connection with the risk management via the social capital leading to a reduction of transaction costs. The impact of these entrepreneurs on the local development for the home Country and the host Country is evaluated; strategies for adapting to the risk. of crowding out of the market are also revealed, because of the recent competition from the Chinese immigrants trading networks.

\section{Introduction}

Le dynamisme entrepreneurial plus marqué chez certaines communautés, notamment chez les immigrants, procède souvent des réseaux sociaux. Ceuxci sont analysés comme des institutions de coordination économique, parallèlement au mécanisme des prix et d'autorité, et qui reposent sur le phénomène de confiance fondée sur des liens de proximité culturelle, professionnelle et spatiale. Étant donné l'ampleur des risques commerciaux et extra commerciaux prévalant dans les pays en voie de développement (PVD) surtout ceux d'Afrique, l'entrepreneur, souvent à la tête d'une très petite entreprise (TPE) ou PME familiale, doit en général faire face à des conditions d'insécurité contractuelle qui l'exposent, du fait de l'asymétrie d'information, à l'opportunisme des partenaires. Dès lors, pour réduire ses coûts de transaction, l'entrepreneur « ethnique » en situation d'immigrant aura intérêt à intégrer ses activités dans les réseaux sociaux de sa communauté (familiale, ethnique, religieuse) dont les règles de fonctionnement sont autant de contraintes pour les membres. De plus, contrairement au reste de l'Afrique, à notre connaissance, il n'existe que de rares travaux sur l'entrepreneuriat d'immigrants en Afrique centrale, en particulier les immigrants originaires du continent; dès lors cette

\footnotetext{
${ }^{a}$ Docteur en économie et HDR, Centre de recherche et d'études prospectives (CREP), Université M. Ngouabi de Brazzaville, Congo

${ }^{\mathrm{b}}$ Docteur en sciences de gestion, NIMEC, IAE-Université de Caen Normandie et ESGAE
} 
étude voudrait combler cette lacune. De plus, nous ciblons les immigrants maliens, car ils forment le groupe le plus visible des milieux d'affaires ouestafricains installés au Congo.

À l'instar d'autres pays, le Mali est soumis aux contraintes de la mondialisation dont l'une des caractéristiques est la forte dynamique migratoire. À cet égard, 4 millions de Maliens vivent à l'étranger (dont 3,5 millions d'immigrés en Afrique; 200000 en Europe); ainsi la diaspora représente plus du quart de la population totale du Mali, selon le rapport du ministère des Maliens de l'extérieur (2016). Le montant annuel de devises transféré par cette diaspora malienne vers le pays d'origine a été estimé à 300 milliards de francs CFA (FCFA) en 2005, dont 120 milliards, soit plus d'un tiers, proviennent de France (Jeune Afrique Intelligent $\mathrm{n}^{\circ} 2370$ du 11 au 17 juin 2006), et ce même rapport indique que le montant des transferts de cette diaspora a représenté $11 \%$ du PIB en 2012. En 2009, selon le FMI (rapport n ${ }^{\circ} 11 / 38$ de février 2011), le montant des transferts de la diaspora malienne vers le pays d'origine a atteint les 174 milliards de FCFA. Sur le continent africain, les migrants maliens sont concentrés dans les pays de la CEDEAO, dans le cadre d'une émigration de proximité, spécialement en Côte-d'Ivoire; en Afrique centrale, on les retrouve aussi dans les pays de la CEEAC, notamment au Gabon, au Cameroun en Angola et au Congo-Brazzaville. En l'état actuel de notre information, il ressort que Brazzaville serait l'une des destinations les plus ouvertes pour les migrants maliens et ceci, depuis les années 1960 à nos jours. En effet, selon le Consulat général du Mali à Brazzaville, on dénombrerait actuellement plus de 23600 immigrés maliens au Congo-Brazzaville, dont 12000 à Brazzaville. La majorité de ces immigrants sont des entrepreneurs qui dirigent des PME familiales. Parmi les ressortissants ouest-africains, les Maliens forment le groupe à plus forte visibilité au Congo, par rapport aux autres groupes (Mauritaniens, Sénégalais, Béninois, Guinéens et autres). Ces immigrants ouest-africains opèrent surtout comme commerçants et, dans une moindre mesure, comme artisans(bijouterie d'or et d'argent, maroquinerie, couture), au point d'avoir exercé une domination dans les activités de distribution et le commerce de détail de biens manufacturés importés, de la décennie 1960 jusqu'au début des années 2000, ceci du fait de la concurrence exercée depuis par l'implantation des réseaux marchands de nouveaux immigrants entrepreneurs venus d'Afrique et d'Asie (réseaux camerounais, libanais, indopakistanais et surtout chinois) au CongoBrazzaville (Devey Malu-Malu, 2015).

Ainsi, en partant de la littérature disponible sur l'entrepreneuriat immigré et ethnique (Martins et Portes, 1980; Paturel, 2004; Cross et Waldinger, 1997; Menzies, Brenner et Filion, 2003; Bonacich, 1973; Costes, 1994; Perreault, Brenner, Menzies, Filion et al., 2007; Levy-Tadjine, 2004; Landa, 1993; Brenner, Ramangalahy, Filion et al., 2000, etc.), ainsi que d'une enquête effectuée en 2010, à Brazzaville, auprès de 220 répondants parmi les entrepreneurs immigrants maliens de plusieurs générations et divers groupes ethniques, cet article cherche à savoir dans quelle mesure, à l'instar d'autres communautés au dynamisme entrepreneurial reconnu et opérant déjà en Afrique centrale (Libanais, Indiens, Mauritaniens, Bamilékés, et plus récemment les réseaux chinois), les performances entrepreneuriales d'immigrants maliens, exerçant au Congo-Brazzaville, par l'intermédiaire des TPE et PME familiales, procèdent-elles de la dynamique du capital social? Celui-ci se traduit par un fort degré de réseautage des ressources ethniques dans le sens que le conçoivent Menzies, Brenner et Filion (2003). Nous avançons l'hypothèse principale que les entrepreneurs immigrants maliens, implantés au Congo depuis les années 1960, et qui sont surtout des commerçants et dans une moindre mesure des artisans, se rapprocheraient d'une "minorité intermédiaire durable » (Bonacich, 1973). En ce sens, outre la focalisation sectorielle dans les activités intermédiaires, spécialement le commerce de détail généré parles «boutiques de quartiers", ce groupe culturel particulier de confession musulmane considère son immigration au Congo, quoique vieille de plus d'un demi-siècle, comme un exil temporaire et tend à résister à l'assimilation au profit d'un repli communautaire qui se traduit par une forte solidarité intracommunautaire (participation aux associations du réseau communautaire, etc.). L'étude empirique identifie les facteurs de succès de cet entrepreneuriat d'immigrants maliens en lien avec la gestion du risque par le recours au capital social qui induit une réduction des coûts de transaction sur le marché, du fait des externalités positives des réseaux; tout en montrant leur mix dans les stratégies managériales d'adaptation au risque fort crédible d'éviction du marché du pays d'accueil. Ceci, du fait de la concurrence récente que leur imposent les réseaux marchands et financiers 
d'immigrants chinois en cours d'implantation depuis 2000, autant au Congo-Brazzaville qu'ailleurs en Afrique centrale.

\section{Gestion du risque par l'entrepreneuriat des immigrants et l'apport du capital social : revue de la littérature}

Dans cette revue de littérature, nous montrons d'abord en quoi la réduction des coûts de transaction est une préoccupation majeure pour l'entrepreneur opérant en réseaux; ensuite, nous établissons l'impact du capital social sur les performances des entreprises d'immigrants.

\subsection{La réduction des coûts de transaction, objectif ultime pour l'entrepreneur opérant en réseaux dans les PVD}

Plus que dans les pays développés, du fait notamment d'un risque pays plus élevé, l'entrepreneur (immigrant ou non) opérant dans les PVD, souvent dans le cadre de PME familiales, est exposé à une grande insécurité contractuelle. Celle-ci est accentuée par la prééminence du secteur informel dans nombre de PVD, spécialement africains, car, selon Hernandez (1996), parmi les coûts de l'informalité figurent les coûts de transaction liés à l'absence de sécurité contractuelle dans l'univers informel. En effet, les contrats légalement exigibles rendent crédibles les promesses faites et incitent les parties à ne prendre que des engagements qu'elles sont susceptibles d'honorer. Ils peuvent même inclure une clause pénale condamnant à une compensation la partie qui déclare forfait. Or, un système légal assurant à tous un accès aisé aux tribunaux facilite les transactions entre agents. Même quand ces garanties existent parfois pour les entreprises du secteur formel, elles restent souvent plus théoriques qu'effectives; d'où la persistance de l'insécurité juridique pour les investisseurs à cause du plus faible niveau de capital social. Ce concept est défini de plusieurs manières par les auteurs (Coleman, 1988; Fukuyama, 1995; Healy et Côté, 2001; Putnam et al., 1995), mais ces derniers s'accordent sur un point commun : le facteur "confiance » qui confère au capital social un caractère d'actif public spécifique à un groupe ou une société. En effet, le facteur « confiance» s'avère un élément clé dans l'élaboration des ré- seaux, quelle que soit la base (famille, ethnie, religion, partenariats d'affaires) sur laquelle ils se constituent. Mais, dans les réseaux professionnels, toute forme de confiance entre managers n'engendre pas ipso facto la performance de leurs organisations. Dans ce sens, si la confiance émanant de la raison ou de la «tête » est positivement associée avec les liens relatifs aux ressources économiques et à l'orientation de la carrière, en revanche la confiance émanant de l'affection ou du «cœur» est positivement associée avec les liens d'amitié et d'orientation de la carrière, mais négativement associée aux liens relatifs aux ressources économiques (Chua, Ingram et Morris, 2008). Dès lors, soulignent ces auteurs, il faut interpréter prudemment comment le facteur confiance, dans le cadre des réseaux professionnels, peut expliquer les performances managériales. Ainsi, définissant le capital social en relation avec l'efficacité des organisations, on peut relever que pour Putnam et al. (1993), le capital social se réfère aux faits de l'organisation sociale, tels que la confiance, les normes et les réseaux, qui sont susceptibles de promouvoir l'efficacité de la société. De même, Menzies, Brenner, Filion et al. (2003) ont établi en quoi le capital social appréhendé à travers le réseautage des ressources ethniques (en matière de main-d'œuvre, de marchés, de fournisseurs, de source de financement) conduirait au succès des affaires pour les entrepreneurs de certaines minorités ethniques à travers le monde.

Dans le même registre, selon Healy et Côté (2001), par exemple, le concept de capital social désigne l'existence de réseaux d'individus partageant des normes, des valeurs et des convictions communes. Son moteur est la confiance; son mode d'exercice est la coopération, le travail en équipe, le partage des savoirs; les lieux de son accumulation étant d'abord la famille, puis la communauté, enfin les institutions publiques et privées. Pour Collier et Gunning (1999), le capital social est conjointement généré par le gouvernement et la communauté. Le capital social public renvoie à la mise en œuvre d'institutions gouvernementales pour la promotion du secteur privé, alors que le capital social civil procède d'avantages économiques émanant de l'interaction sociale qui découle pour l'essentiel, d'un développement des affaires dans la confiance, d'une réduction des coûts de transaction et d'une meilleure connaissance des externalités de réseaux sociaux. Ces auteurs constatent un plus faible niveau de capital social dans les pays africains par 
rapport aux autres PVD, du fait d'une plus grande corruption, d'une bureaucratie plus tatillonne et d'une plus importante fragmentation de la société découlant de la grande diversité ethnolinguistique.

Dès lors, en présence de marchés imparfaits, marqués par l'asymétrie d'information, l'opportunisme des agents et une régulation institutionnelle (au sens de capital social public) absente ou inefficace, l'entrepreneur des PVD sera de plus en plus exposé à l'insécurité contractuelle, synonyme de risques et induisant divers coûts de transaction, en particulier les coûts de recherche des partenaires, ceux liés à la négociation, ainsi que les coûts résultant de la nécessité de faire respecter les contrats. Dans cette optique, Williamson (1975) a précisé le concept de coûts de transaction en distinguant d'une part, les coûts de transaction ex ante liés à la sélection du contractant, à l'activité de rédaction, de négociation et de protection d'un accord; d'autre part, les coûts de transaction expost, tenant aux nécessaires ajustements au coursde perturbations non anticipées et à l'évaluation des comportements opportunistes (coûts d'administration, de surveillance et de contrôle mis en place par les partenaires pour veiller au respect des clauses contractuelles, coûts de marchandage et de renégociation découlant des corrections, coûts de structure liés au comportement de passagers clandestins, coûts de retrait d'un partenaire). En conséquence, face à cette faiblesse de dispositifs juridiques chargés de faire respecter les contrats, l'entrepreneur, pour pallier ces insuffisances du marché, se trouve contraint d'intégrer ses activités dans une logique de réseaux sociaux reposant sur le mécanisme de la confiance. Ainsi, en l'absence d'une régulation efficace ou suffisante, émergent ipso facto des modes de régulation basés sur des réseaux sociaux particularistes à dominante communautaire (familiale ou clanique, ethnique, religieuse), en vue de sécuriser les transactions marchandes. Historiquement, on doit à North (1991) cette idée du rôle déterminant que jouent les institutions (au sens des règles ou normes informelles, à savoir : coutumes, codes de conduite, traditions; ainsi que des règles formelles, c'est-à-dire : constitutions, lois, droits de propriété) dans la sécurisation des transactions pour une meilleure coordination marchande. Il note que :

Throughout history, institutions have been devised by human beings to create order and reduce uncertainty in exchange. Together with the standard constraints of economics they define the choice set and therefore determine transaction and production costs and hence the profitability and feasibility of engaging in economic activity (North, 1991, p. 97).

Ainsi, à côté des trois institutions de coordination des activités de l'entreprise identifiées par Williamson (le marché et son corollaire le contrat, l'entreprise verticalement concentrée, les relations contractuelles suivies), Landa (1993) soutient que le réseau particulariste ethno-familial serait une forme d'organisation dominante dans les PVD. Pour lui, le réseau ethnique apparaît comme un quatrième mode d'organisation économique dans lequel un rapport de confiance réciproque relie les parties contractantes. Ce type de réseau parviendrait à protéger ses membres au regard des conditions d'insécurité contractuelle et des contextes historique et institutionnel qui distinguent les PVD. Ce faisant, de tels réseaux sociaux contribuent sensiblement, pour les entrepreneurs, à la réduction de l'incertitude et des risques autant commerciaux qu'extra commerciaux et partant, à la réduction des coûts de transaction. Àcet égard, il convient de noter en substance trois points :

- la confiance réciproque entre parties rend moins coûteux l'effort de respect des engagements, en indiquant le degré de distance sociale, l'identité de parenté ou d'appartenance communautaire; elle transmet aussi un signal permettant de déterminer si le partenaire éventuel est ou non digne de confiance, sans qu'il soit nécessaire de consacrer des frais importants à la recherche de l'information;

- la confiance réciproque entre deux maillons d'une chaîne d'acteurs dans le milieu d'affaires entraîne une confiance transitive qui réduit la nécessité de se renseigner sur la réputation de tel ou tel autre partenaire indirect;

- l'existence d'un dense réseau d'informations, fruit des associations d'entraide caractérisant le groupe ethnique, dispense l'entrepreneur du devoir coûteux de s'assurer à l'aide de ses propres ressources de la bonne réputation d'un partenaire potentiel. 


\subsection{Performances d'immigrants entrepreneurs et capital social}

Le dynamisme entrepreneurial des communautés d'immigrants a souvent été expliqué en se référant au capital social, grâce à l'accès aux ressources du groupe ethnique, dans ce sens que les entrepreneurs membres de communautés dont la cohésion sociale est plus forte disposent d'un avantage comparatif sur les autres dans la conduite de leurs affaires. La littérature anglo-saxonne en ce domaine distingue souvent deux courants théoriques: la théorie de l'enclave ethnique et la théorie des minorités intermédiaires.

\section{La théorie de l'enclave ethnique (ethnic enclave theory)}

Martins et Portes (1980) ont montré qu'une communauté ethnique peut promouvoir les activités dans une enclave complexe, avec ses propres usines manufacturières, ses magasins de gros et de détail, ses réseaux d'affaires dans les services, ses PME qui contrôleraient les conditions de concurrence; à l'instar de ce qui a été établi pour la réussite de l'enclave intégrée parmi les immigrants cubains de Miami. L'existence de telles enclaves ou niches exige la présence d'immigrants dotés d'assez de capitaux et savoir-faire entrepreneurial, ainsi que l'offre régulière d'une main-d'œuvre émanant d'une immigration durable, à l'enclave. Dans cette optique, c'est essentiellement le faible coût de la main-d'œuvre immigrée qui permet la survie et l'expansion des entreprises de l'enclave, tout en favorisant la création de nouvelles occasions pour le développement de l'enclave. Dans ce sens, Paturel (2004) relève que pour faire face à leurs difficultés et à d'éventuelles discriminations, la stratégie la plus courante pour les migrants serait l'appui sur les réseaux ethniques et sur les occasions évoquées plus haut. Ainsi, une niche se caractériserait au terme du processus et sur un espace géographique donné, par une forte homogénéité ethnique avec une forte hétérogénéité sociale. Autrement dit, par association d'une forme d'entrepreneuriat immigré tourné vers la communauté ethnique et de réseaux d'embauche ethnique (Cross et Waldinger, 1997), ce phénomène traduirait la mise en place d'une division du travail -intra-ethnique limitant les échanges avec le reste de la société d'accueil. Cette pratique de l'enclave serait typique de l'intégration de la diaspora chinoise dans les pays développés comme en France ou aux États-Unis. En revanche, dans les PVD, comme dans les pays d'Afrique centrale où l'immigration d'entrepreneurs chinois (commerçants) est récente et ne date que des années 2000, leurs réseaux d'affaires s'intègrent davantage à la société d'accueil, surtout pour l'embauche de la main-d'œuvre, même si dans leurs boutiques ne sont vendus que des produits made in China. Ici comme en Asie de l'Est, les immigrés chinois ont tendance à s'intégrer dans le modèle des minorités intermédiaires. Nous verrons plus loin que les réseaux marchands et financiers chinois constituent actuellement la concurrence la plus redoutable pour d'autres immigrants entrepreneurs opérant en Afrique centrale, tels les réseaux d'immigrants maliens du Congo-Brazzaville.

\section{La théorie des minorités intermédiaires}

(middleman minority man)

Elle a été développée par Bonacich (1973) et Bodell (1980) et elle repose sur le concept de minorité intermédiaire qui procède d'un groupe culturel particulier occupant une position intermédiaire dans la société d'accueil, laquelle position résulte d'un avantage compétitif par rapport à une forte capacité d'adaptation. Deux conditions doivent être satisfaites par ces minorités: d'abord considérer leur migration comme un exil temporaire et partant, avoir le projet de retourner dans leur pays d'origine, d'où une résistance à l'assimilation et un repli communautaire qui se traduit par une forte solidarité intra-ethnique (création de leurs propres associations professionnelles, culturelles, pratique de l'endogamie, etc.). Ceci tend à induire une hostilité de la part de la société d'accueil. Cette théorie a été appliquée pour expliquer l'entrepreneuriat des Juifs ashkénazes émigrés en Europe centrale, des Chinois en Asie du Sud-Est, des Arméniens de Turquie, des Asiatiques en Afrique orientale et en Afrique australe et des Libanais d'Afrique de l'Ouest et d'Afrique centrale. Leur immigration, étant vécue comme un exil temporaire mais à l'issue incertaine, justifierait, soulignent Paturel et LevyTadjine (2004), le confinement sectoriel à des activités intermédiaires, en particulier le commerce de détail par le fait qu'il s'agit des secteurs où l'entrée et la sortie sont assez aisées (capital de départ modeste, facilité de revente en cas de retour au pays). Cependant, bien souvent, relèvent les mêmes auteurs, le retour souhaité n'a pas lieu, soit parce que les conditions politiques, ne le permettent pas, soit parce que les entrepreneurs immigrés estiment ne 
pas encore disposer d'épargne suffisante pour s'y établir, soit paradoxalement parce qu'ils sont victimes de leur succès dans leur pays d'accueil et qu'ils ont conscience qu'ils ne connaittraient pas la même réussite économique dans leur pays d'origine. Dans ce cas, en devenant « minorité intermédiaire durable», les entrepreneurs immigrés ont deux options possibles : soit cultiver le mythe du retour et de rester dans une logique ethnique en refusant toute forme d'assimilation, soit renoncer au rêve de la patrie et s'installer dans le nouveau pays (Levy-Tadjine, 2004). Ceci passe, selon Bonacich (1973), par des signes forts tels que l'adhésion à des associations non ethniques, l'exogamie, l'emploi de personnes d'ethnies différentes, etc. En revanche, les immigrés correspondant à ce profil attachent une grande importance à l'éducation de leurs enfants qui, fréquemment, quittent l'économie ethnique et s'intègrent plus rapidement dans la société d'accueil.

Dans le cas d'entrepreneurs immigrants d'origine malienne opérant à Brazzaville, il semblerait qu'on soit en présence d'une "minorité intermédiaire durable ». Plusieurs arguments viennent en appui à cette affirmation: étant donné leur présence ancienne sur plusieurs générations et leur assez bonne intégration économique et sociale depuis la fin des années 1960 dans la société d'accueil dont ils parlent couramment les langues véhiculaires (kikongo et lingala), leur projet de retour au Mali (PIB per capita de $692 \$$ USD en 2010, puis 830 \$ en 2016 selon le FMI), classé PMA, reste hypothétique du fait notamment qu'ils considèrent le Congo-Brazzaville (PIB per capita de 2983 \$ en 2010, puis 1784 \$ en 2016 selon le FMI), pays pétrolier et à revenu intermédiaire, comme un eldorado pour leurs affaires et comme leur seconde patrie. De plus, certains de ces ressortissants ont épousé une femme congolaise et donc acquis la nationalité du pays d'accueil. Néanmoins, à l'instar d'autres minorités intermédiaires, les immigrants maliens ont tendance à récuser tout processus d'assimilation à la société congolaise, en préservant leur mode de vie de la culture musulmane dans un pays majoritairement chrétien et en refusant d'adhérer à des associations professionnelles extracommunautaires.

Le capital social venant en appui à l'entrepreneuriat, comme le soulignent Perreault, Brenner et al. (2007), il ressort de ces deux courants théoriques que le dynamisme de l'entrepreneuriat d'immigrants s'explique pour l'essentiel par l'accès aux ressources du réseau communautaire ou ethnique en tant que source de financement, de main-d'œuvre bon marché, d'approvisionnement, d'information et de conseils, mais aussi en tant que marché. La littérature en ce domaine distingue deux niveaux d'analyse (Brenner, Filion et al., 2000); le premier concerne le démarrage de l'entreprise et le second renvoie à l'explication du succès des entreprises d'immigrants. S'agissant du premier niveau, on soutient d'abord que nombre d'entrepreneurs des groupes ethniques sont eux-mêmes issus de milieux familiaux et culturels valorisant l'esprit d'entreprise; ensuite, la communauté ethnique constitue la première source de soutien et d'occasions d'affaires à laquelle l'entrepreneur immigrant se trouve souvent exposé. En outre, les recherches ont établi qu'en général, la création d'entreprises est centrée sur le réseau familial et intra-ethnique. Le réseau présenterait plusieurs avantages pour l'entrepreneur : accès à un bassin d'investisseurs potentiels et d'entrepreneurs expérimentés; accès à une maind'œuvre bon marché; possibilité pour l'entrepreneur d'opérer dans sa langue maternelle et son milieu social et culturel, ce facteur étant amplifié par le fait que les immigrants recourent peu aux réseaux et services gouvernementaux d'appui du pays d'accueil. S'agissant du second niveau d'analyse, l'accès aux ressources du groupe ethnique constitué est un des principaux déterminants du succès d'immigrants entrepreneurs. En effet, les immigrants ne disposant pas du capital initial pour la création de l'entreprise obtiendront le financement grâce à des prêts consentis au sein de leur communauté, par des institutions, des relations ou de la famille. De plus, étant donné que le succès exige au départ de longues heures de travail souvent peu rémunérées, la main-d'œuvre bon marché émanant de la famille de l'entrepreneur constitue une ressource critique au maintien des activités. De plus, le réseau serait aussi un moyen de protection contre la discrimination faite au groupe ethnique dans le pays d'accueil et vis-à-vis de la compétition. Il représenterait en outre un moyen facilitant la communication avec la société d'accueil et l'acquisition de connaissances en gestion.

En résumé, ce réseautage de l'entrepreneuriat d'immigrants procède du capital social et Menzies, Brenner et Filion (2003) ont établi en quoi le capital social appréhendé parle réseautage des ressources 
ethniques en matière de main-d'œuvre (coethnicemployees), de marchés (co-ethnicmarkets), de fournisseurs (co-ethnicsuppliers); de source de financement (co-ethnic sources of finance), conduirait au succès des affaires pour les entrepreneurs de certaines minorités ethniques à travers le monde. De même, Perreault, Brenner, Menzies, Filion et al. (2007), qui ont analysé la relation entre le capital social et la performance de l'entrepreneuriat ethnique en contexte canadien, ont établi en substance que l'appartenance de l'entrepreneur à des associations communautaires (sociales, d'affaires, ou professionnelles) est positivement corrélée avec la performance de l'entreprise.

\section{Résultats de l'enquête sur les entrepreneurs immigrants maliens opérant au Congo-Brazzaville}

Cette partie empirique présente d'abord la méthodologie de l'enquête et les caractéristiques sociodémographiques des répondants. Ensuite, nous analysons l'impact du capital social sur le dynamisme entrepreneurial d'immigrants échantillonnés; enfin, nous décrivons la gamme des secteurs d'activités des répondants avant d'en déduire leurs stratégies managériales d'adaptation à la concurrence des réseaux marchands et financiers chinois en pleine émergence au Congo.

\subsection{Méthodologie de l'enquête et caractéristiques sociodémographiques des répondants}

Les données de terrain ont été obtenues sur la base d'une enquête effectuée en 2010 dans le cadre du projet de recherche "Entrepreneuriat des immigrants en Afrique centrale » que nous supervisons au CREP depuis plus de cinq ans. Pour ce faire, un questionnaire a été élaboré et soumis à 220 répondants. Ce questionnaire comporte cinq rubriques permettant de saisir la dynamique entrepreneuriale d'immigrants maliens au Congo. Le protocole d'enquête comportait des questions portant sur les caractéristiques sociodémographiques; les motifs d'immigration vers le Congo-Brazzaville et la province d'origine; le mode de financement de l'activité menée dans le pays d'accueil et le management de la succession; l'impact de leurs activités sur le pays d'origine et le pays d'accueil; enfin les questions relatives au réseautage de leurs activités ont permis d'appréhender le niveau de capital social des répondants selon, grosso modo, la démarche de Menzies, Brenner et Filion (2003) que nous avons adaptée au contexte local, à savoir le recours aux ressources du réseau ethnique en matière de maind'œuvre, de source de financement, de fournisseurs. De même, nous avons tenté de mesurer le capital social des répondants par rapport à leur participation aux associations à caractère social (mutuelles des originaires de tel district ou telle région du Mali), culturel (confréries religieuses) ou professionnel (tontines, corporations des bijoutiers, couturiers, commerçants, etc.) de la communauté.

Pour faciliter la communication avec les répondants (dont la majorité ne parle qu'un « français approximatif»), nous avons retenu un étudiant d'origine malienne qui a donc pu réaliser les interviews dans la langue malienne, le bambara, couramment pratiquée par les immigrants ainsi qu'en français pour les rares répondants ayant un niveau d'éducation secondaire ou supérieur. En outre, le fait que notre enquêteur soit étudiant à la maitrise en économie à l'Université Marien Ngouabi de Brazzaville, et fils d'un diplomate malien qui a été en poste à Brazzaville, lui a conféré une influence supplémentaire pour vaincre les réticences et outrepasser les règles de confidentialité qui caractérisent en général les communautés d'immigrants, spécialement ceux qui pratiquent les affaires dans le pays d'accueil. Après avoir distribué 300 questionnaires aux individus cibles, dans les circonscriptions administratives, à savoir les sept arrondissements de Brazzaville où exercent les entrepreneurs maliens (spécialement les quatre plus anciens arrondissements qui comptent chacun un grand marché et une mosquée: Poto-Poto, Ouenzé, Bacongo, Moungali), nous avons enregistré un retour de 220 documents exploitables, soit un taux de réponse de $73 \%$. De plus, pour une meilleure représentativité de notre échantillon nous avons appliqué la méthode des quotas combinée au choix aléatoire des individus cibles par rapport au domaine d'activité et à la localisation de celle-ci dans Brazzaville. Enfin, le dépouillement des données a été effectué sur le logiciel SPSS.

Les caractéristiques sociodémographiques des répondants ont été étudiées en fonction des critères 
suivants : situation matrimoniale, lieu de résidence des épouses, niveau d'études et l'ethnie d'origine.

- Au regard de la situation matrimoniale, il ressort de notre enquête que 85 répondants sont mariés, soit $38 \%$ du total, contre 65 entrepreneurs vivant en union libre $(29,5 \%)$ et le même pourcentage de célibataires. Le reste, soit $3 \%$, est divorcé. L'union libre concerne les répondants qui ont choisi de cohabiter avec une femme congolaise; celle-ci en général est de religion chrétienne, tandis que son mari est musulman. Quant au lieu de résidence des épouses, il ressort que plus de la moitié de répondants, à savoir 45 entrepreneurs (soit $52 \%$ sur un total de 85 mariés) résident avec leurs épouses au Congo. Les autres ont soit leur épouse résidant au Mali $(28 \%$ ) soit une épouse dans le pays d'origine et dans le pays d'accueil (20\%). Dans ce dernier cas, il s'agit donc d'entrepreneurs polygames qui sont en général les plus fortunés de la communauté d'immigrants maliens.
- Concernant le niveau d'instruction, l'enquête indique que la majorité des répondants $(60 \%)$, soit 132 sur un total 220, sont soit analphabètes soit n'ont suivi que des études primaires. Les autres ont le niveau du secondaire $(30 \%)$ et du supérieur $(10 \%)$. Cette situation reflète les tendances bien connues de la diaspora d'origine malienne dans le monde qui comporte majoritairement une main-d'œuvre faiblement qualifiée et qui est occupée soit comme ouvriers dans les pays développés soit comme commerçants ou artisans dans les PVD notamment en Afrique de l'Ouest et en Afrique centrale.

- S'agissant de l'ethnie d'origine des entrepreneurs immigrants, il ressort du tableau 1 cidessous que l'ethnie Sarakolé (30\%) serait le groupe le plus important; viennent ensuite l'ethnie Peul $(20 \%)$ et l'ethnie Bambara $(19,5 \%)$. La question de l'origine ethnique étant politiquement sensible en Afrique, en particulier parmi les immigrants, on comprend que près d'un cinquième des entrepreneurs visités (18\%) ait refusé d'y répondre.

\begin{tabular}{|l|c|c|}
\hline \multicolumn{1}{|c|}{ Nom du groupe ethnique } & Effectif & Pourcentage \\
\hline Sarakolé & 66 & 30 \\
\hline Peul & 44 & 20 \\
\hline Bambara & 43 & 19,5 \\
\hline Diogoramé & 20 & 9 \\
\hline Malinké & 9 & 4 \\
\hline Refus de répondre & 38 & 18 \\
\hline Total & 220 & 100 \\
\hline
\end{tabular}

Tableau 1 - Groupe ethnique d'origine des entrepreneurs immigrants de Brazzaville Source : Notre enquête, Brazzaville, 2010

Enfin, sur les mobiles de leur immigration vers le Congo, les entrepreneurs échantillonnés, à titre principal, ont avancé à concurrence de $95 \%$ (210 sur 220 répondants) des raisons socioéconomiques : soit améliorer leur situation économique et celle de leur famille restée dans le pays d'origine, soit par incitation volontaire à entreprendre des affaires à l'étranger, soit encore pour répondre à l'appel d'un parent déjà installé dans le pays d'accueil. Plus spécifiquement, le reste des répondants, soit $5 \%$, nous ont affirmé qu'en tant qu'anciens éleveurs appartenant surtout au groupe ethnique des Peuls, ils ont dû fuir la sécheresse des 
années 1970 qui avait décimé leur cheptel de bovins au Mali, ainsi que les conflits armés sévissant au nord du pays depuis les années 1990, et se sont reconvertis en entrepreneurs immigrants, notamment dans l'artisanat. En outre, même si la majorité des répondants ont refusé de donner leur âge par souci de confidentialité, nous avons constaté cependant que les immigrants visités appartiennent à plusieurs générations et que leur âge se situerait dans la fourchette de 25 ans pour les plus jeunes à 65 ans voire 70 ans pour les plus âgés. Ces derniers, en général dans leur troisième âge, cèdent la succession managériale et patrimoniale de leur entreprise au profit de leur fils.

\subsection{Réseautage des activités et dynamisme de l'entrepreneuriat d'immigrants maliens}

Pour tenter de mesurer l'impact du capital social sur l'entrepreneuriat d'immigrants maliens, nous avons tenté d'appliquer la démarche de Menzies, Brenner et Filion (2003) déjà évoquée plus haut, que nous avons adaptée au contexte local.

- En matière de source de financement. 94 entrepreneurs échantillonnés, soit $43 \%$ du total, ont financé leur capital initial par un apport personnel; les autres, soit $57 \%$, ont recouru aux ressources du réseau ethnique (famille et communauté) pour démarrer leurs affaires. Comme nous pouvons le voir, aucun répondant n'a sollicité un appui financier aux organismes publics ou privés locaux, car en général les immigrants affichent un comportement de réserve et de méfiance à l'égard de tels organismes du pays d'accueil. De même, après le démarrage de leurs activités, le financement du haut du bilan comme du bas du bilan se fait surtout par autofinancement $(55 \%)$ et par emprunt auprès du réseau de la communauté (25\%); à peine $20 \%$ ont eu recours au crédit auprès des institutions financières du pays d'accueil, spécialement les institutions de microfinance; mais aussi auprès de ECOBANK une banque commerciale à participation majoritaire d'actionnaires ouestafricains qui s'est récemment implantée au Congo sous forme de banque filiale. En effet, comme l'a constaté Lévy-Tadjine (2004), la faible propension à l'emprunt bancaire est due, chez les entrepreneurs immigrants, à la fois aux tracasseries administratives dont les opérateurs économiques font l'objet, mais aussi à des anticipations de discrimination au crédit; d'autant que les immigrés sont souvent peu instruits et sont porteurs de projets de taille réduite, en général dans le commerce de détail (boutiques).

- En matière de fournisseurs. 140 répondants, soit près des deux tiers de notre échantillon, déclarent avoir comme principaux fournisseurs, pour leurs approvisionnements en marchandises, d'autres entrepreneurs maliens exerçant dans le négoce ou l'import-export, y compris parla pratique d'achats groupés au sein de réseaux marchands de leur communauté. Les autres $(25 \%)$ traitent avec des fournisseurs d'autres nationalités étrangères, notamment les ressortissants du pays voisin, le Congo-Kinshasa (surtout pour la livraison d'or destiné aux artisans bijoutiers de Brazzaville), ainsi que ceux originaires de Chine, France, Thailande, Dubaï et des Émirats arabes unis. De ce fait, moins de $10 \%$ des répondants ont déclaré s'approvisionner auprès des fournisseurs congolais, à l'occasion de leurs activités. On constate ainsi un réseautage important dans les circuits d'approvisionnement d'immigrants maliens opérant au Congo-Brazzaville, ce qui est conforme au comportement managérial en ce domaine des minorités intermédiaires dans les pays d'accueil comme pour les réseaux marchands chinois, indiens ou libanais d'Afrique centrale ou d'ailleurs.

- En matière de main-d'œuvre. Le réseautage apparaît aussi important, car notre enquête révèle que 98 répondants, soit $45 \%$ de notre échantillon, bénéficient de l'apport de la maind'œuvre d'un proche parent ou de la communauté, la part de la main-d'œuvre d'origine communautaire dans l'emploi total étant toujours supérieure à $65 \%$ pour ces entrepreneurs immigrants. De plus, pour les autres entreprises de l'échantillon, soit $55 \%$, il s'agit en fait souvent de micro entreprises unipersonnelles, à savoir les boutiques de quartier pratiquant le commerce de détail, surtout dans l'alimentation et les matériaux de construction. Dès lors, comme nous l'avons dit plus haut, le recours à la main-d'œuvre bon marché et mobilisable au-delà de la durée hebdomadaire légale du travail dans le pays d'accueil, constitue une ressource critique au maintien des activités, voire explique notoirement les performances des entrepreneurs migrants dans le contexte du Congo-Brazzaville où les réseaux 
marchands d'immigrants ouest-africains (Maliens, Mauritaniens, Sénégalais, Guinéens, Béninois), contrôlent le petit commerce de proximité par l'entremise des boutiques localisées aux abords des grands marchés ainsi que dans les divers quartiers. Ainsi dans leurs boutiques de quartier, il est courant qu'après avoir ouvert dès $7 \mathrm{~h}$ du matin, la fermeture ne survienne que vers $22 \mathrm{~h}$ voire minuit, tandis que les boutiques tenues par les Congolais ferment plus tôt, en général vers $20 \mathrm{~h}$ du soir.

En outre, en matière de participation aux associations ou clubs du réseau communautaire, l'enquête a aussi révélé un niveau élevé de capital social en ce sens que tous les répondants $(100 \%)$ ont reconnu appartenir à au moins l'une des associations à caractère socioculturelle et professionnelle créées par les immigrants maliens du Congo-Brazzaville (y compris les institutions islamiques de microfinance).Il s'agit notamment de l'Association des ressortissants du District de Bamako en République du Congo-Brazzaville, de l'Association des ressortissants de la région de Kayes, de Koulikoro, de Ségou, de Sikasso et de l'Association des ressortissants du Grand Nord qui regroupe les immigrants originaires de Mopti, Tombouctou et Gao.

Nous avons aussi établi que c'est dans le cadre de ces associations que les membres organisent leurs tontines selon des rapports de proximité relationnelle, professionnelle et culturelle et coordonnent leurs stratégies pour contribuer au développement local de leur pays d'origine. Ce résultat est conforme à celui trouvé par Perrault, Brenner, Menzies et al. (2007) dans le contexte de l'entrepreneuriat d'immigrants au Canada, où il apparaît une forte corrélation positive entre le capital social mesuré notamment par la participation des entrepreneurs aux associations du réseau communautaire et la performance des entreprises. Par contre, il est apparu dans notre enquête que le réseau d'immigrants maliens ne pourrait pas être considéré comme un débouché significatif pour les répondants, étant donné que pour l'essentiel ces derniers ciblent le marché extracommunautaire, à l'exception d'immigrants opérant dans certains services, en particulier la restauration.

Enfin, le réseau ethnique particulariste étant aussi un cadre approprié d'apprentissage et de transmission de l'esprit d'entreprise, notre enquête a établi que chez les immigrants entrepreneurs maliens, dirigeants d'entreprises familiales, la succession en affaires (sur le double plan managérial et patrimonial) est assurée en priorité au profit du fils. Ce type de management de la succession, synonyme d'une meilleure pérennité des PME familiales, est couramment observé dans d'autres communautés dont la diaspora manifeste un dynamisme entrepreneurial. C'est le cas des entrepreneurs du groupe Bamiléké du Cameroun ou des entrepreneurs du groupe Nandé au Congo-Kinshasa (RDC), ou encore des entrepreneurs indiens qui opèrent depuis longtemps en Afrique centrale, sous le modèle de minorité intermédiaire. L'enquête nous enseigne que la majorité des répondants préparent leur succession en affaires au profit de leur fils en l'associant à la gestion de l'entreprise familiale dès l'âge de la majorité. L'enquête nous a permis de constater que pour les entrepreneurs qui ont déjà atteint le troisième âge alors que leurs fils sont encore mineurs, le management de la succession consiste à associer aux activités de l'entreprise familiale, le fils mineur aux parents proches et adultes du réseau familial (frère, neveu) afin de le préparer à prendre la relève le moment venu. En l'absence d'un fils candidat à la succession, l'entrepreneur prépare de préférence son frère cadet pour la relève. Mais en général, c'est le fils qui a la préférence de nos répondants en matière de succession en affaires (53\%).

\subsection{Domaines d'activité des immigrants maliens et stratégies managériales d'adaptation à la concurrence des réseaux marchands et financiers chinois}

Le tableau 2 révèle que la majorité des répondants opèrent dans le secteur des services. Les propriétaires de boutiques se classent au premier rang avec $50 \%$. Ces boutiques sont situées autour des grands marchés de Brazzaville (Bacongo, Ouenzé, Poto-Poto, Moungali) ainsi que dans les quartiers des sept communes que compte Brazzaville, dans le cadre d'un commerce de proximité avec la clientèle. Notre enquête indique que la quincaillerie serait le deuxième domaine d'activité pour les immigrants maliens. Il s'agit là d'un créneau porteur, car depuis les années 2000, avec le boom pétrolier, le BTP connait une expansion rapide au Congo et les classes aisées de la population investissent massivement dans l'immobilier. En résumé, les immigrants maliens sont surtout des commerçants $(90 \%)$ et accessoirement des artisans $(10 \%)$ dans les domaines suivants : couture, bijouterie, maroquinerie, vannerie. 


\begin{tabular}{|l|c|c|}
\hline \multicolumn{1}{|c|}{ Type d'activité } & Effectif & Pourcentage \\
\hline Boutiques(alimentation/tissus/parfumeries) & 110 & 50 \\
\hline Quincaillerie(matériaux de construction, etc.) & 44 & 20 \\
\hline Électroménagers et TIC & 22 & 10 \\
\hline $\begin{array}{l}\text { Pièces détachées auto et vente de motos } \\
\text { Autres services (restauration, pressing, } \\
\text { cybercafés, bureautique) }\end{array}$ & 15 & 7 \\
\hline $\begin{array}{l}\text { Artisanat (couture, vannerie, bijouterie, } \\
\text { maroquinerie) }\end{array}$ & 6 & 3 \\
\hline \begin{tabular}{l} 
Total \\
\hline
\end{tabular} & 23 & 10 \\
\hline
\end{tabular}

Tableau 2-Domaines d'activité des immigrants maliens au Congo-Brazzaville Source : Notre enquête, Brazzaville, 2010

Avec l'implantation récente des réseaux marchands et financiers chinois, les commerçants maliens et autres groupes d'immigrants ouest-africains doivent faire face depuis les années 2000 à une sévère concurrence qui les conduit à s'adapter à la nouvelle donne au risque d'être menacés par le phénomène d'éviction du marché du pays d'accueil. Ce risque d'éviction est d'autant plus crédible que l'implantation des commerçants chinois bénéficie du statut de grand pays émergent qu'est la Chine ainsi que de l'appui des pouvoirs publics de Pékin et de Brazzaville. En effet, lors de la visite du président Denis Sassou Nguesso en Chine en 2000, un accord sur la promotion et la protection des investissements fut signé; c'est en vertu de cet accord que les commerçants chinois ont été autorisés à ouvrir leurs boutiques au Congo à compter de 2005. De plus, dans le cadre du partenariat stratégique liant le Congo avec la Chine, depuis 2006, il est prévu que le fonds gouvernemental China-Africa Development Fund doté de 5 milliards de dollars US contribue à l'appui financier des investisseurs chinois en Afrique, y compris au profit des commerçants chinois. Nous avons de bonnes raisons de penser que cette situation devrait durer dès lors que les commerçants chinois installés en Afrique constituent un tremplin essentiel pour les exportations de biens manufacturés vers les marchés africains, par les groupes industriels opérants, spécialement dans les zones économiques spéciales (ZES) en Chine et qui sont incités par l'État chinois à conquérir les débouchés extérieurs. Les immigrants chinois installés au Congo sont actuellement estimés à 7000 personnes selon Park (2009); en dehors du commerce général, ils exercent dans divers domaines d'activité comme employés des FMN chinoises opérant dans le pétrole, BTP, les TIC, les cimenteries, l'exploitation forestière et minière, ainsi que les PME sous contrôle familial qui opèrent dans la santé (les cliniques privées et les salons de beauté), la restauration et l'hôtellerie, ainsique l'agriculture et l'exploitation forestière. Les immigrants maliens ont déjà perdu d'importantes parts de marché dans le pays d'accueil pour les créneaux de distribution comme les tissus, la parfumerie, la quincaillerie, l'électroménager et les TIC (téléphones portables et accessoires), les produits plastiques et en porcelaine (en particulier, les ustensiles de cuisine) à cause d'une meilleure compétitivité prix en faveur des commerçants chinois. En effet, les immigrants chinois qui vendent exclusivement des marchandises made in China pratiquent des prix fort avantageux, en moyenne $30 \%$ à $50 \%$ plus bas que leurs concurrents. Cette compétitivité prix s'explique notamment par des conditions d'approvisionnements dont bénéficient les immigrants chinois opérant en Afrique (Dupre et Shi, 2008). Ainsi, ces commerçants chinois ont développé des réseaux commerciaux privilégiés et stables avec leur pays d'origine. Ils sont en contact direct avec les producteurs de marchandises; ceci leur permet d'éviter les intermédiaires, ce qui réduit d'autant leur coût d'approvisionnement et préserve leur marge commerciale. De plus, les commerçants chinois bénéficient souvent de relations privilégiées avec leurs fournisseurs; il 
s'agit en général d'un lien entre filiales et la maison mère ou par l'entremise des différentes branches d'une entreprise familiale.

En résumé, ces caractéristiques de l'approvisionnement des immigrants chinois, à savoir : contact direct, exclusivité et stabilité dans les relations avec les fournisseurs du pays d'origine, expliquent la réussite économique des commerçants chinois au Congo et ailleurs sur le reste du continent. Cependant, les immigrants visités tentent de s'adapter à cette nouvelle donne qui les expose à un réel risque d'éviction du marché du pays d'accueil. On constate déjà depuis ces dernières années que plusieurs boutiques appartenant à des commerçants maliens et qui étaient situées autour des quatre grands marchés de Brazzaville ont été rachetées par les immigrants chinois. De plus, la principale avenue commerciale de Brazzaville, l'avenue de la Paix, située au centre-ville et jouxtant deux communes à forte implantation d'immigrants ouestafricains, serait déjà occupée à plus de $30 \%$ par des commerçants chinois et pourrait devenir dans un futur proche le Chinatownde Brazzaville. Plus généralement en Afrique, comme le relève Cabestan (2013), les immigrants entrepreneurs chinois concurrencent parfois directement les acteurs économiques locaux, en particulier dans les secteurs de la distribution et du petit commerce. L'immigration chinoise s'est accélérée depuis les années 2000 dans la majorité des pays africains, notamment dans les pays pétroliers et miniers d'Afrique centrale comme l'attestent les statistiques du tableau 3.

\begin{tabular}{|l|c|c|}
\hline \multicolumn{1}{|c|}{ Pays } & $\begin{array}{c}2001 \text { (Ohio University data } \\
\text { base) }\end{array}$ & Estimation \\
\hline Angola & 500 & $20000-40000$ (en 2006) \\
\hline Burundi & - & $150 \quad$ (en 2007) \\
\hline Congo-Brazzaville & - & 7000 (en 2006) \\
\hline Congo-Kinshasa & 200 & 10000 (en 2007) \\
\hline Cameroun & 50 & $1000-7000$ (en 2005) \\
\hline $\begin{array}{l}\text { Gabon } \\
\text { Tchad }\end{array}$ & - & 6000 (en 2008) \\
\hline $\begin{array}{l}\text { Total d'immigrants chinois en } \\
\text { Afrique (dont Afrique du Sud) }\end{array}$ & 129605 & $300-500$ (en 2008) \\
\hline
\end{tabular}

Tableau 3 - Limmigration chinoise en Afrique centrale pour la période 2001-2008

Source : Park Yoon Jung (2009). Chinese migration in Africa. Occasional Paper, 24(janvier), 3. Repéré à www.saiia.org.za

Comme stratégies d'adaptation à cette concurrence, les immigrants interviewés ont tendance à privilégier celles qui mobilisent les facteurs de compétitivité hors prix, notamment en proposant le service après-vente et une meilleure garantie à leur clientèle pour des produits importés de Chine à des conditions d'approvisionnement moins avantageuses (d'où l'apparition d'ateliers de réparation et dépannage d'appareils électroménagers, motos, téléphones portables). L'autre stratégie en cours d'émergence est la recherche de produits de substitution importés d'autres pays émergents. Ces produits coûtent un peu plus cher que les produits made in China, mais sont considérés comme plus fiables et donc de meilleure qualité. C'est le cas surtout des produits de consommation durable et de biens intermédiaires tels que les électroménagers et les TIC, les matériaux de construction et les biens d'équipements, importés des pays suivants : Corée du Sud, Inde, Indonésie, Singapour, Malaisie, Taïwan, Brésil. Mais ces immigrants s'approvisionnent aussi, à titre 
accessoire, dans d'autres pays développés, surtout au Japon pour les produits de l'électronique grand public. À notre avis, c'est le développement de cette seconde stratégie couplée avec la promotion des activités artisanales (couture, bijouterie, maroquinerie) dont les Maliens détiennent un savoir-faire reconnu depuis des décennies, qui pourrait assurer la survie d'immigrants entrepreneurs maliens et autres réseaux marchands ouest-africains au Congo-Brazzaville. Une dernière stratégie constatée chez ces immigrants consiste à se positionner dans une division du travail avec les commerçants chinois, comme demi-grossistes pour servir d'intermédiaires entre eux et les détaillants congolais. Mais à terme, cette dernière stratégie est condamnée à l'échec étant donné que la réglementation commerciale en vigueur, au nom du « patriotisme économique », privilégie les Congolais pour l'exercice du commerce de demi-gros et de détail.

Enfin, s'agissant de l'impact des immigrants sur le développement local du pays d'origine et du pays d'accueil, notre enquête indique que 205 immigrants maliens, soit $93,2 \%$, procèdent plus ou moins régulièrement à l'envoi de fonds aux membres de leurs familles dans le pays d'origine (en moyenne 60000 FCFA ou 100 USD par transfert mensuel). Les canaux de transfert privilégiés sont les sociétés de transfert de fonds (Western Union et Money Gram) et, dans une moindre mesure, les canaux informels. Ainsi pour les immigrants, les transferts financiers constituent une forme de protection sociale ou d'assurance-vie par rapport aux incertitudes et à la précarité sociale des parents résidant dans le pays d'origine. Étant donné la défaillance de services étatiques de sécurité sociale, ces transferts contribuent à la réduction de la pauvreté et au renforcement des capacités individuelles et institutionnelles permettant aux populations de participer au processus de développement local. En outre, au regard de la contribution au développement local de leur pays d'origine, il ressort de l'enquête que 96 Maliens, soit 43,6 \% de notre échantillon, participent au développement local de leur pays d'origine parle financement de projets de développement communautaire. Les domaines privilégiés sont la construction d'écoles, de centres de santé ou encore le forage de puits pour l'eau potable. Par rapport à la région d'origine des immigrants, il ressort de notre enquête que Koulikoro et Kayes sont les deux régions qui reçoivent le plus d'appui de la diaspora malienne installée au Congo pour la réalisation de projets de développement communautaire et local.
En effet, sur un total de 220 répondants, 41 originaires de Koulikoro, soit 18,6 \%, et 35 originaires de Kayes, soit 15,9\%, déclarent avoir participé au moins une fois à ce type de projet, en tant qu'immigrants entrepreneurs opérant au Congo. De même, les immigrants maliens contribuent au développement local du pays d'accueil parle paiement régulier d'impôts et taxes, la création 'emplois et le transfert de savoir-faire surtout dans les métiers de l'artisanat; sans oublier les contributions de leurs associations professionnelles et culturelles aux projets communautaires (projets sanitaires et culturels).

\section{Conclusion}

Les minorités dites «intermédiaires 》 font généralement face à de l'hostilité dans le pays d'accueil, notamment en période de crise politique et économique; on les considère comme des "boucs émissaires", c'est-à-dire un groupe social exutoire cristallisant le mécontentement populaire, parfois avec le soutien de gouvernements en déficit de légitimité. Le principal reproche qui est fait à ces minorités intermédiaires durables dans les pays d'accueil concerne justement leur «réussite dans les affaires", comme l'a souligné Bourgi (2005) pour le cas des immigrants entrepreneurs libanais d'Afrique de l'Ouest et d'Afrique centrale. Cette situation s'est matérialisée, en particulier dans des pays d'accueil dirigés par des pouvoirs non démocratiques, par des émeutes, des mouvements d'exclusion et d'expulsion (plus de 50000 Indiens furent expulsés d'Ouganda en 1972 lors de l'opération dite de « dé-indianisation » par le régime dictatorial du président Idi Amin);des Libanais régulièrement accusés de contrebande de diamants, puis l'expropriation massive des hommes d'affaires portugais, grecs, libanais et autres en 1973 lors de la «Zaïrianisation » sous le régime dictatorial du président Mobutu en RDC; les expulsions massives de commerçants ouest-africains du Congo-Brazzaville sous le régime militaire du président Yhomby-Opango en 1978. Les minorités intermédiaires se heurtent à la discrimination de la société d'accueil dans la mesure où les deux communautés ont parfois des intérêts divergents. En effet, ces immigrants peuvent susciter l'opposition des entrepreneurs nationaux qui se sentent menacés par la réussite économique des immigrants accusés de pratiquer une concurrence déloyale. De même, l'organisation efficace des réseaux marchands et financiers d'immigrants rend souvent la participation des nationaux quasi impossible, dans certaines activités, comme le commerce, dans un environnement concurrentiel; 
ceci engendre des réactions discriminatoires. Les entrepreneurs immigrants maliens, comme nous l'avons montré, attestent une certaine réussite économique au Congo-Brazzaville grâce à un niveau élevé de capital social dans le management de leurs activités, au sein de PME familiales.

Nous avons mesuré l'impact du capital social sur l'entrepreneuriat de ces immigrants en reprenant la démarche de Menzies, Brenner et Filion (2003) que nous avons adaptée au contexte du milieu d'affaires congolais. Nous avons établi un haut niveau de réseautage des activités du groupe d'immigrants maliens en matière de main-d'œuvre, de source de financement, de fournisseurs, ainsi que de participation des entrepreneurs investigués aux associations professionnelles et culturelles du réseau communautaire. Mais il est apparu dans notre enquête que le réseau d'immigrants maliens ne pourrait pas être considéré comme un débouché significatif pour les répondants, étant donné que pour l'essentiel ces derniers ciblent le marché extra communautaire. Par ailleurs, les immigrants maliens participent dans une certaine mesure au développement socio-économique du pays d'accueil en ce sens, par exemple, qu'ils s'acquittent régulièrement de leurs obligations fiscales, créent de l'emploi et transfèrent au profit des Congolais un savoir-faire ou know-how managérial, spécialement dans les domaines du commerce et de l'artisanat. Enfin, devant la montée de la concurrence que leur imposent depuis le milieu de la décennie 2000 les réseaux marchands et financiers chinois, nous avons montré que les immigrants maliens tentent de relever le défi en déployant des stratégies entrepreneuriales qui privilégient la compétitivité hors prix pour éviter le risque d'éviction du marché dans le pays d'accueil.

Une des pistes de recherche que l'enquête suggère reviendrait à mener une étude comparative du comportement entrepreneurial de plusieurs groupes d'immigrants originaires du Sud, dans le contexte des pays d'Afrique centrale (Ouest-Africains, Libanais, Indiens, Chinois), dans le prolongement de Kamavuako (2009). Ceci permettrait d'affiner l'analyse de la dimension stratégique, à savoir celle liée à la pérennisation de l'émergence organisationnelle; d'où un approfondissement des aspects tels que la spécialisation sectorielle des activités des immigrants par groupe cible ou le management de la succession dans les entreprises qui sont souvent des PME familiales, ou encore le rôle du capital social extracommunautaire dans le succès entrepreneurial des immigrants; ainsi que la question de la motivation principale de l'entrepreneuriat immigré au regard des occasions génériques du marché.

\section{NOTES}

1 Cet article est la version actualisée d'une communication présentée au XIe Congrès international francophone en entrepreneuriat et la PME, tenu à Brest du 24 au 26 octobre 2012 à l'Université de Bretagne Occidentale. Les auteurs remercient les referees anonymes du XIe CIFEPME, ainsi que ceux de la revue Organisations et territoires pour leurs précieux commentaires et suggestions, néanmoins ils restent seuls responsables d'éventuelles erreurs subsistantes.

\section{RÉFÉRENCES}

Bonacich, E. et Bodell, J. (1980). The economic basic of ethnic solidarity: Small business in the Japan American community.Berkeley, CA: University of California Press.

Bonacich, E. (1973).A theory of middleman minorities. American Sociological Review, 38(4), 583-594.

Bourgi, A. (2005). Libanais en Afrique, ou d'Afrique? Outre-Terre, 11, Revue européenne de géopolitique, 11, 149-153.

Brenner, G.A., Ramangalahy, L. et Filion, J. (2000). Entrepreneuriat ethnique et rôle des réseaux de soutien : les entrepreneurs chinois dans trois grandes villes canadiennes. Cabier de recherche,2000-09(3), 1-13.

Cabestan, J.P. (2013). Les relations Chine-Afrique : nouvelles responsabilités et nouveaux défis d'une puissance mondiale en devenir. Hérodote, 150(3), 150-171.

Chua, R.Y., Ingram, P. et Morris, M.W. (2008).From the head and the heart: Locating cognition and affect-based trust in managers'professional networks. Academy of Management Journal, 51(3), 436-452.

Coleman, J. S. (1988). Social capital in the creation of human capital. American Journal of Sociology, 94(suppl.), 95-120.

Collier, P. et Gunning, J.W. (1999). Explaining African economic performance.Journal of Economic Literature, 37(mars), 65-68. 
Costes, L. (1994). La dimension ethnique : une explication du comportement économique. Revne française de sociologie, 35(2), $231-249$.

Cross, M. et Waldinger, R. (1997, septembre). Intégration économique et évolution du marché du travail, Communication présentée à la II ${ }^{e}$ Conférence internationale, Metropolis, Copenhague. Repéré à www.international.metropolis.net/research. policy/econf/

Devey Malu-Malu, M. (2015, 14 août). Congo-Brazzaville : les Ouest-Africains, rois du commerce. Jeune Afrique. Repéré à www.jeuneafrique.com

Dzaka-Kikouta, T., Makany, R.A et Kamavuako, J. (2005). Capital social et entrepreneuriat des immigrants en Afrique centrale : cas des Angolais opérant en RDC.Revue congolaise de gestion, 9-10(2), 55-78.

Dzaka-Kikouta, T. (2003). Formation à la culture entrepreneuriale et identité ethnique au Congo-Brazzaville chez les entrepreneurs Bakongo : une analyse par la confiance et les réseaux sociaux. Dans M. Niculescu, et B. Ponson (dir.), La formation à l'entrepreneuriat (p. 89-104) Paris, France : AUF. Repéré à www.entrepreneuriat.auf.org

Dupre, M. et Shi, W. (2008). La présence chinoise en Afrique de l'Ouest. Le cas du Mali et du Bénin. Document de travail, 2008-69, 1-42. Repéré à www.afd.org

Fukuyama, F. (1995). Social capital and the global economy. Foreign Affairs, 75(5), 89-104.

Healy, T. et Côté, S. (2001). Le capital social : indispensable mais insaisissable. Problèmes économiques,2717(13 juin), 23-29.

Hernandez, E.M. (1996). L'entrepreneur informel africain et le concept de gestion éco-sociale. Notes de recherche du Réseau entrepreneuriat de l'AUF, 96-54, 15-16.

Kamavuako Diwavova, J. (2009). Problématique de l'entrepreneuriat immigré en République démocratique du Congo, essai de validation d'un modèle (Thèse de doctorat inédite). Université de Reims.

Landa, J.T. (1993). Culture et activité entrepreneuriale dans les pays en voie de développement : le réseau ethnique, organisation économique. Dans Berger et al. (dir.),Esprit d'entreprise, cultures et sociétés (p. 208-209) Paris, France : Maxima-Laurent du Mesnil.

Levy-Tadjine, T. (2004). L'entrepreneuriat des immigrés et son accompagnement en France (Thèse de doctorat). Université du Sud Toulon-Var.

Menzies T.V., Brenner, G. A. et Filion, L.J. (2003). Social capital, networks and ethnic minority entrepreneurs: Transnational entrepreneurship and bootstrap capitalism. Dans H. Etemadet R.W. Wright (dir.), Globalization and entrepreneurship: Policy and strategy perspectives (p.125-151). Northampton, MA: Edward Elgar, McGill International Entrepreneurship Series.

Ministère des Maliens de l'extérieur (2016, juin). Guide des Maliens de l'extérieur, 1-134.

North, D. C. (1991). Institutions. Journal of Economic Perspectives, 5(1), 97.

Perreault, C., Brenner, G.A, Menzies, T.V., Filion, L. J. et Ramangalahy, C. (2007). Social capital and business performance: Ethnic enterprises in Canada.International Journal of Business and Globalisation, 2(1), 145-160.

Park Yoon Jung (2009). Chinese migration in Africa. Occasional paper, 24(janvier), 3. Repéré à www.saiia.org.za

Paturel, R. et Levy-Tadjine, T. (2004, mars). Entrepreneuriat immigré et accompagnement de projets :construire des ponts Nord-Sud par l'entrepreneuriat immigré. Actes du XVe Colloque annuel du CEDIMES, Alexandrie.

Putnam, R.D et Helliwell, J.F. (1995).Economic growth and social capital in Italy. Eastern Economic Journal, 21(3), $295-307$.

Putnam, R.D., Leonardi, R. et al. (1993). Making democracy work: Civic traditions in modern Italy. Princeton, NJ: Princeton University Press.

Waldinger, R. (1989). Structural opportunity or ethnic advantage? Immigrant business development in New York. Migration Review, 23(1) 48-72.

Waldinger, R., Aldrich, H. et al. (1990).Ethnic entrepreneurs: Immigrants business in industrial societies, Newbury Park, CA: Sage Publications.

Williamson, O.E. (1975). Market and hierarchies, analysis and antitrust implications. New York, NY: New York Free Press. 